\title{
COMPARATIVE STUDY OF THE PERMEABILITY INDUCED BY AROMATIC AND NON AROMATIC HEPTAENES ON SMALL AND LARGE LIPIDIC VESICLES
}

\author{
C. M. Gary-Bobo and B. Cybulska* \\ Service de Biophysique, Département de Biologie, CEN Saclay \\ 91191 Gif-Sur-Yvette Cedex, France \\ *Départment of Pharmaceutical Technology and Biochemistry \\ Technical University, Gdansk, Poland
}

(Received for publication December 14, 1981)

\begin{abstract}
The efficiency in inducing cation permeability of vacidin $A$, candicidin $D$ and amphotericin $\mathrm{B}$ has been compared on small and large lipidic vesicles. Amphotericin B is two times less effective on large than on small vesicles. On the contrary, vacidin $\mathrm{A}$ and candicidin $\mathrm{D}$ are about ten times more effective on large than on small vesicles. These data are consonant with what is observed on living cells.
\end{abstract}

In a previous report ${ }^{11}$, it has been shown that aromatic heptaenes vacidin $\mathrm{A}$ and candicidin $\mathrm{D}$ were slightly less effective than the non aromatic heptaene amphotericin B in inducing cationic permeability on small sonicated lipidic vesicles, at variance with what is constantly observed on living cells, in which aromatic heptaenes are 10 to 100 fold more effective than non aromatic one ${ }^{2)}$.

This discrepancy between living cells and sonicated vesicles has been attemptively ascribed to the very large difference in size between the two systems: on small vesicles, the permeability measurements would have to be related to the number of polyenic molecules necessary to make a single pore per vesicle. On living cells the same permeability measurements would reflect rather the intrinsic properties of the many pores being present at a time in the membrane.

In order to check this hypothesis, the efficiencies of amphotericin B on one hand and vacidin A and candicidin D on the other, have been measured comparatively on small sonicated unilamellar vesicles (SUV) and on large unilamellar vesicles (REV) obtained by reverse phase evaporation of the same lipidic composition and under the same experimental conditions by the method of electroneutral proton-cation exchange previously described ${ }^{1}$. The results obtained demonstrate that, the efficiency of aromatic heptaenes is much greater than the efficiency of amphotericin B on large vesicles, although this is not the case on small ones. This finding supports the hypothesis made previously and may account for the observations made on living cells.

\section{Materials and Methods}

L- $\alpha$-Phosphatidylcholine was prepared from egg yolk according to Patel and Sparrow ${ }^{3)}$. Phosphatidic acid was enzymatically prepared from it. Cholesterol was purchased from Fluka, FCCP (carbonyl cyanid- $\alpha$-trifluoromethoxyphenyl hydrazone) from Boehringer, amphotericin B was from Squibb, whereas vacidin $\mathrm{A}$ and candicidin $\mathrm{D}$ were isolated and purified in the Department of Pharmaceutical Technology, Technical University, Gdansk (Poland).

Both types of vesicles were prepared with the same mixture of lipid: L- $\alpha$-phosphatidylcholine, phosphatidic acid and cholesterol in a molar ratio 70: 10:20. 
Sonicated unilamellar vesicles (SUV) were prepared as described previously ${ }^{1)}$ using $30 \mathrm{mg}$ of lipid mixture per $\mathrm{ml}$ of a $400 \mathrm{~mm}$ sodium phosphate buffer, $\mathrm{pH} 5.20$.

Large unilamellar vesicles (REV) were prepared according to SzOKA and PAPAHADJOPOULOs ${ }^{4}$ using $30 \mathrm{mg}$ of lipid mixture per ml of $200 \mathrm{~mm}$ sodium phosphate buffer, pH 5.20.

The experiments were carried out in the following conditions:

SUV: $500 \mu$ l of the stock suspension were diluted in $4.5 \mathrm{ml}$ of isotonic sodium sulfate, adjusted to $\mathrm{pH}$ 7.40. Twenty $\mu 1$ of a $10^{-2} \mathrm{M}$ FCCP in ethanol was added and then the polyene, as microliter amounts of a freshly made solution in dimethyl formamide. The final lipid concentration in the titrating vessel was about $3 \mathrm{mg} / \mathrm{ml}$.

REV: Fifty $\mu \mathrm{l}$ of the stock suspension were diluted in $5 \mathrm{ml}$ of sodium sulfate, adjusted at $\mathrm{pH}$ 7.40. Then $20 \mu \mathrm{l}$ of FCCP were added and the polyene as before. The final lipid concentration was about $0.3 \mathrm{mg} / \mathrm{ml}$. In this condition, approximately the same amount of proton was available in both SUV and REV samples, in spite of a 10 fold difference in lipid concentration. Finally it must be pointed out that REV are osmotically active (SUV are not). Since the osmolality of the phosphate buffer increases with $\mathrm{pH}$, the osmolality of the external medium was continuously adjusted during the proton efflux through a proper adjustment of the osmolality of the titrating $\mathrm{NaOH}$ solution, in such a way that the internal and external medium were isotonic throughout the whole experiment.

\section{Results}

It has been shown in preliminary experiments, that the proton-cation exchange method was applicable to REV as well as to SUV: 1) In absence of any ionophore the proton efflux, practically undetectable on SUV, amounts to about 3 to $5 \%$ per hour of the total titratable proton on REV. This basic flux remains negligible as compared to the proton flux elicited by polyene addition, which develops in 5 to 10 minutes, but at the lowest concentrations, in which case it has been taken into account. 2) FCCP added alone is not able to promote any significant increase of this basic flux. 3) In presence of the highest concentrations of polyenes the proton efflux mediated by FCCP is maximum above $10^{-5} \mathrm{M}$ on SUV and $2 \times 10^{-8} \mathrm{M}$ on REV. 4) The total amount of titratable proton in the sample is measured after Triton X100 addition. The intravesicular volume calculated on this basis was found equal to $1.1 \pm 0.2 \%$ of the $30 \mathrm{mg}$ lipid/ml stock suspension of SUV and $20 \pm 2 \%$ of the $30 \mathrm{mg} \mathrm{lipid} / \mathrm{ml}$ stock suspension of REV. 5) Under the combined influence of FCCP and high concentration of polyene, $95 \sim 98 \%$ of this total amount of proton were titratable on SUV, $83 \sim 87 \%$ on REV, indicating a larger proportion of multilamellar vesicles. Although these multilamellar vesicles could be removed by filtration on $0.2 \mu$ Nucleopore filter, this procedure was not used because of the undesired effect of vesicular size reduction.

The results obtained are given in Figs. $1 \sim 3$. In these figures, the amount of proton which can be released under the action of the polyene is expressed in $\%$ of the maximum amount released at high polyene concentration. The concentration of polyene is expressed in moles per mole lipids. In Fig. 1 are compared the sensitivity of SUV (black dots) and REV (open circles) to amphotericin B. There is no important difference between the two systems; however it is worth noting that the sigmoidal curve is steeper in the SUV case, (from $10^{-4}$ to $10^{-3} \mathrm{M} / \mathrm{M}$ ), than in the REV case which expand from $10^{-4}$ to $10^{-2} \mathrm{M} / \mathrm{M}$. As a result, it appears that this polyene is more efficient in SUV than on REV. The crosses represent the results obtained on uncharged SUV (L- $\alpha$-phosphatidylcholine 80, cholesterol 20 mole \%, no phosphatidic acid); the effect of charge is practically unsignificant. Such a comparison cannot be made on REV, since uncharged REV are very unstable and aggregate rapidly.

In Figs. 2 and 3 are given the corresponding results for vacidin A and candicidin D respectively. In the case of the two aromatic polyenes a large difference is observed between SUV and REV: the 
Fig. 1. Amphotericin B dose-response curve on SUV ( ) REV $(\bigcirc)$ and uncharged SUV $(+)$.

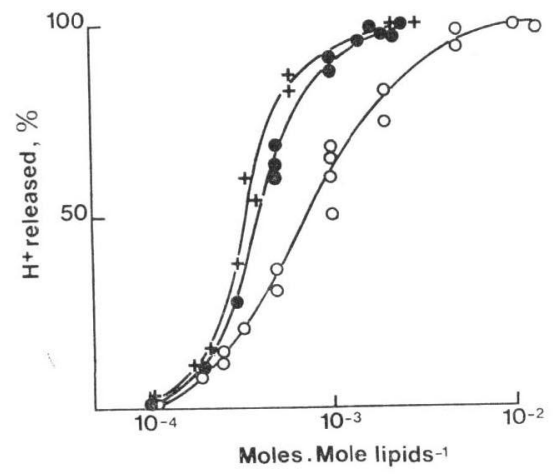

Table 1. Moles polyenes/mole lipids necessary for $50 \%$ proton release on SUV and REV.

\begin{tabular}{l|rrr}
\hline & Amphotericin B & Candicidin D & Vacidin A \\
\hline SUV & $4 \times 10^{-4}$ & $3.5 \times 10^{-3}$ & $1.8 \times 10^{-3}$ \\
REV & $7 \times 10^{-4}$ & $4.5 \times 10^{-4}$ & $1.3 \times 10^{-4}$ \\
\hline
\end{tabular}

efficiency is about 10 times greater on REV than on SUV. Here again the effect of electric charge on SUV is not significant (not shown). The relative efficiency of polyene on these two vesicles taken as the polyene concentration, in moles per mole of lipids, necessary to obtain the release of $50 \%$ of their proton are given in Table 1 .
Fig. 2. Candicidin D dose-response curve on SUV (•) and REV (O).

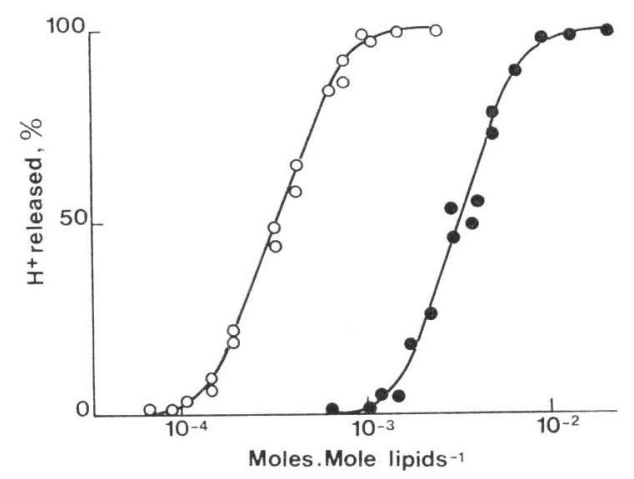

Fig. 3. Vacidin A dose-response curve on SUV and REV $(O)$.

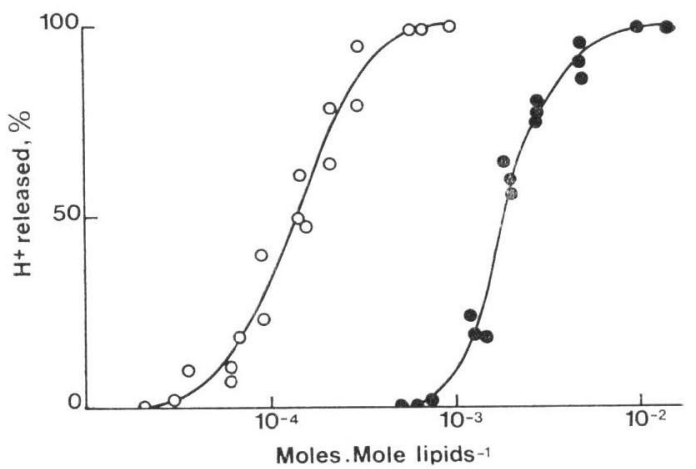

\section{Discussion}

It appears from Table 1 that amphotericin B is nearly 2 times less efficient on REV than on SUV and that the two aromatic heptaenes are about 10 times more efficient of REV than on SUV. These results altogether confirm the results previously obtained on SUV ${ }^{1)}$ and that the size of the vesicle is an important parameter to consider when comparing the efficiencies of pore-forming antibiotics such as polyenes on model systems.

The large vesicles obtained by reverse phase evaporation, REV, are rather heterogeneous in size as compared to SUV; their diameter ranges from 500 to $5000 \AA^{4)}$. However a reasonable estimation of $2500 \AA$ can be made, that is a mean diameter 10 times greater than SUV. This corresponds to a one hundred fold difference in the surface and approximately in the number of lipid molecules per vesicle and a difference much larger yet in volume (theoretically a thousand fold if a strictly spherical shape is assumed).

On this basis, and using the figures of Table 1, a rough calculation of the number $\mathrm{N}$ of polyene molecules per vesicles can be made: on SUV, it comes out that 1 amphotericin B molecule, and about 4.5 and 9 molecules of vacidin A and candicidin D respectively are necessary to obtain a $50 \%$ proton release.

On REV, the corresponding $\mathrm{N}$ are about 200 for amphotericin B, 100 for candicidin and only 30 for vacidin A. Assuming that the number of polyene molecules necessary to make one pore is the same in REV than in SUV, two interpretations might be proposed: either the ability to interact with REV and 
SUV is different for aromatic and non aromatic polyenes, or, aromatic heptaene makes pores more efficient than non aromatic ones. This would be observed only on REV, because their internal volume is large enough to make the rate of proton equilibration between internal and external medium a limiting factor, which is not the case on SUV ${ }^{1)}$.

From this view point, large vesicles appear a better model of living cells than small sonicated vesicles. As a matter of fact, the data obtained on REV can account for the data obtained on living cells, which are much more sensitive to aromatic heptaenes than to non aromatic ones. On REV, vacidin $\mathrm{A}$ is about 5 times more efficient than amphotericin B; this difference is not as large as in cells, but cells are much bigger yet than REV.

\section{References}

1) Cybulska, B.; E. Borowski, Y. Prigent \& C. M. Gary-Bobo: Cation permeability induced by two aromatic heptaenes, vacidin A and candicidin D on phospholipid unilamellar vesicles. J. Antibiotics 34: 884 891,1981

2) Hammond, S. M.: Biological activity of polyene antibiotics. Progr. Med. Chem. 14: 104 179, 1977. $c f$. also reference 8, 10 and 12 in Reference 1).

3) Patel, K. M. \& J. T. Sparrow: Rapid large-scale purification of crude egg phospholipids using radially compressed silica gel column. J. Chromatogr. 150: 542 547, 1979

4) SzoKA, Jr., F. \& D. PApahadopoulos: Procedure for preparation of liposomes with large aqueous space and high capture by reverse-phase evaporation. Proc. Natl. Acad. Sci. USA 75: 4194 4198, 1978 